\title{
Gestures as evidence of student thinking about physics
}

\author{
Rachel E. Scherr \\ University of Maryland, College Park MD 20742-4111, rescherr@umd.edu
}

\begin{abstract}
Student gestures are part of how students articulate their ideas, and can be of use to us in diagnosing student thinking and forming effective pedagogical responses. This paper presents examples of gestures that occur in a conversation between students and a TA about a mechanics homework problem, and analyzes one gesture that was particularly significant to the conversation.
\end{abstract}

\section{Introduction}

Gestures, the spontaneous hand or body movements that normally accompany face-to-face conversation, are potentially an important component of interpersonal communication. This paper presents an episode of conversation among physics students and a teaching assistant in which gestures figure prominently in the discussion. My purpose is to illustrate that attention to gestures can be a powerful tool for diagnosing student thinking and forming effective pedagogical responses [1].

\section{Prior research on gesture and learning}

Research on gesture analysis appears in literature from cognitive science, linguistics, and learning sciences, and includes much to interest physics education researchers. Some of the research is potentially of immediate use in diagnosing student thinking. Roth, for example, claims that gestures that substitute for speech may convey ideas that are literally pre-articulate; before people can give precise verbal descriptions of their ideas, those ideas may be revealed by their gestures [2]. Crowder, in observing middle-school students explaining astronomical models, has documented that gestures tend to be interwoven with speech when people are "explaining in the moment;" when students describe something familiar, their gestures redundantly emphasize speech [3]. It is easy to imagine teachers benefiting from this research right away.

Other gesture research has both pedagogical and theoretical implications. Garber and GoldinMeadow offer evidence that gesturing can actually facilitate problem-solving: people "offload" thinking into gesture as into a sketch, freeing up cognitive effort for other tasks [4]. Roth and Lawless go so far as to suggest that the act of gesturing may activate the same neuronal assemblies as the language that goes with the gesture, thereby assisting neurologically with language emergence [5].

Gesture-speech mismatches are a particularly rich field for investigation, as they seem to indicate when students are "of two minds." Church et al observed that children performing Piaget conservation tasks use gestures in their explanations, and that sometimes those gestures contradict their speech (indicating "wider" when the child said "taller," for example). The researchers classified the students as to whether they were conserving or nonconserving, and also as to whether their gestures were "concordant" or "nonconcordant," in a subsequent teaching task, nonconserving nonconcordant children learned the most [6]. In a more recent study, Garber et al. observe that gesture-speech mismatches cluster at strategic choice points in solving the Tower of Hanoi puzzle [4], suggesting that cognitive exploration of more than one solution path is taking place.

\section{"Trajectory" episode}

In this paper, I draw examples of the use of gestures from a conversation among three students (Mike, Robin, and Jenny) working together with a teaching assistant on their mechanics homework [7]. In the minute-and-a-half that I analyze in detail, the students are trying to draw velocity vectors at various points along the trajectory of an object in projectile motion. In particular, the students are discussing whether the velocity of the object is zero at the top of its trajectory. In this case, the velocity of the ob- 


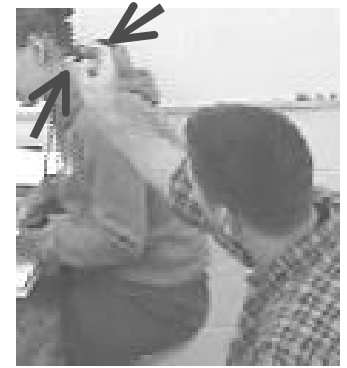

Figure 1. "The one at the top should have zero length?"

(Teaching assistant)

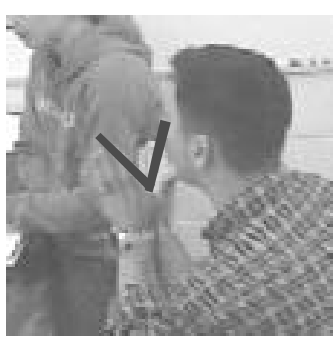

Figure 2. "two diffe rent directions" (Teaching assistant)

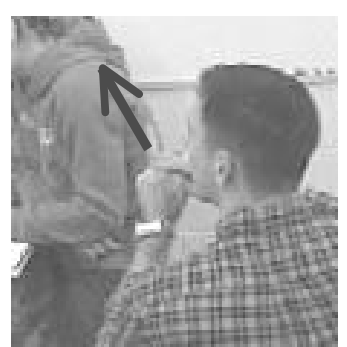

Figure 3. "vectors"

(Teaching assistant)

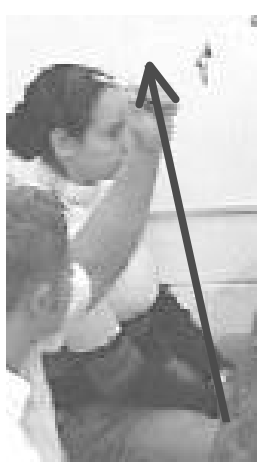

Figure 4. "It's not increasing its ...height" (Mike) ject at the top of its trajectory is horizontal.

In the first thirty-five seconds of the 1.5-minute episode, the students are unsure about the velocity of the object at the top. In the next thirty seconds, the TA guides the students to think about the components of the velocity vectors for the object. At the end of the episode, the group agrees that at the top the object has horizontal velocity, but not vertical. A transcript of the episode follows. (T, J, M, and R are the teaching assistant, Jenny, Mike, and Robin respectively.)

T: And you said the one at the top should have zero length? Is that -

J: We weren't going to draw an arrow.

M:We're not - we're not sure what to do.

J: Since the velocity's zero at that one point

$\mathrm{T}$ : Is it not moving at that point?

J: Right.

T: Why?

J: It kinda comes to a s- - well,

M:Changing direction?

$\mathrm{J}$ : Does it come to a stop at that point? If it stopped

$\mathrm{R}$ : There's one instant when it ha-

J: So it just-

T: Well, let's try this. Let's just try - I mean - to break up the two different directions. I think you've done this in class where you draw, um, vectors in different directions you break them up into components. Have you done that in class? Where you have like a vertical and a horizontal

R: Kinda.

J: I don't know what we've done in class.

M:I'm trying to pick up where you're going with this.

J: Yeah.
T: Okay, where I'm going. If you just think about it moving left to right, does it ever stop when it's moving left to right?

R: No.

T: Why?

J: Wouldn't it just fall straight down then if it was like -Wmp! Psh. [gestures]

M:[Laughs] I don't know.

J: If it stopped and then it - I'm trying to think.

M:It still has I guess horizontal motion, but not...

T: Not vertical.

J: Vertical.

M:It's not increasing it's...height.

J: Right.

\section{Some gestures in the episode}

Jenny, Mike, and the TA all gesture during the episode. In the first line, while saying "the one at the top should have zero length," the TA holds his arm out straight toward the picture of the trajectory that is on the board above the students and holds his index finger and thumb close together, as though to indicate something small (Figure 1). When he suggests thinking about "two different directions," he holds his two hands flat and at right angles to one another (Figure 2). For "vectors," he slides the tip of his right index finger along and then beyond his pointed left index finger (Figure 3). Jenny shrugs at least twice (a gesture analyzed extensively by McNeill [9]), and at the end of the episode Mike shoots his outstretched arm straight upwards while saying "It's not increasing its...[gesture] height" (Figure 4). Other gestures appear in the episode as well. 


\section{The "half-parabola" gesture}

One gesture by Jenny, the only gesture marked in the transcript, is particularly significant to the conversation. As she says "Wouldn't it just fall straight down then if it was like -Wmp! Psh," her left hand moves up in front of her body in a halfparabola ("if it was like"), stops at the vertex ("Wmp!"), and finally drops straight down to the table ("Psh"). Figure 5 is a still frame illustrating the gesture.

One measure of the gesture's significance is that her statement is unintelligible without it. Previous research [2] prompts us to consider the possibility that as we watch Jenny gesture we are watching her idea about the object's motion emerge; the idea is too new to be articulated verbally, but it appears imagistically in the gesture. Other research [3] reinforces our impression that Jenny is "explaining in the moment" rather than describing a familiar idea, since her statement interweaves ge sture and speech.

Another measure of the gesture's significance is that it is an intuitively compelling expression of Jenny's thinking about the motion, and the partic ipants treat it as such; at Jenny's gesture, the TA stops talking, and within a few seconds the group reaches the correct conclusion about the velocity at the top of the trajectory. Finally, the gesture is eloquent; it's hard to imagine words that would complete Jenny's sentence with anything like the clarity and brevity that the gesture provides.

\section{A missed opportunity for diagnosis}

The half-parabola gesture appears at first to function, conversationally, as an answer to the homework question - or at least a strong, commonsense refutation of an answer that was competing with the correct answer. Also, appearing as it does immediately after the TA intervention about components, the gesture seems to be a result of that intervention. It is tempting to conclude that the TA intervention was a helpful one; it seems to have elicited the half-parabola gesture, which gave the students a common-sense basis for the correct response.

However, closer examination of the videotape reveals that the cited occurrence of the half-parabola gesture was not, in fact, its first occurrence. In the first half of the episode, about forty seconds before the event described above, Jenny's hand makes the half-parabola gesture twice as she muses quietly: "If it stopped... so if it stopped..." Jenny's incomplete sentences function as musing to herself (since no

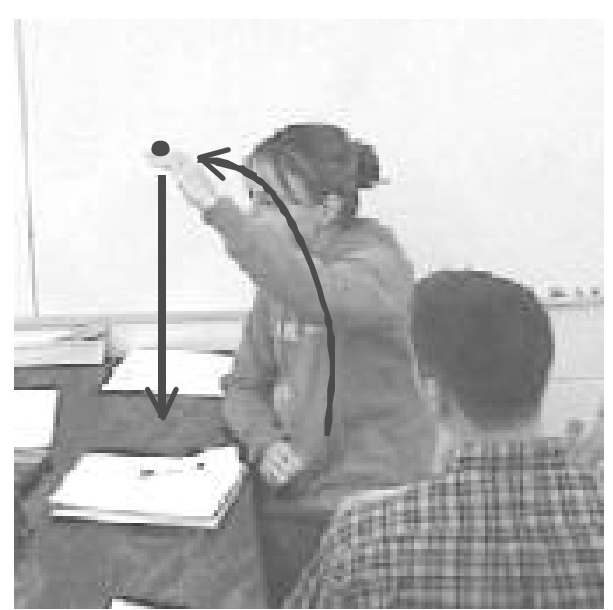

Figure 5. The half-parabola gesture.

one responds to her); the TA overlaps her musing to begin his intervention about components.

Since the gesture first occurred before the TA intervention, it was surely not a result of that intervention - and perhaps the intervention was not as helpful as one might have thought, at least to Jenny. On the contrary, for Jenny, the intervention appears to have been an interruption in her thinking. Closer examination of the video reveals that Jenny appears to be waiting for the TA to finish so that she can speak; just after the initial gestures, she draws a breath as though to begin talking, but then looks at the TA and closes her mouth tightly.

Instructors use many cues to diagnose student thinking. Mostly, we attend to the details of students' spoken or written language. I hope that the above example illustrates that we can greatly benefit from also attending to student gestures. Had the TA in this episode been alert to Jenny's gesture, he might not have drawn her away from her own thoughts with an interjection about components. Instead, he might have inquired about the initial occurrences of the half-parabola gesture, perhaps by imitating and questioning it: "What do you mean by this?"

\section{Conclusion}

This paper has attempted to demonstrate that there are compelling reasons to attend to student gestures while they solve physics problems. The gestures present us with diagnostic opportunities, which, like other diagnostic opportunities, can and should have direct pedagogical consequences.

In addition, I hope to have shown that gesture research literature contains much to interest the physics education researcher. 


\section{References}

1. D. Hammer and D. Schifter, "Practices of inquiry in teaching and research," Cognition and Instruction 19(4) (2001) 441-478

2. W.-M. Roth, "From gesture to scientific language," J. of Pragmatics 32 (2000) 1683-1714

3. E. Crowder, "Gestures at work in sensemaking science talk," J. of the Learning Sciences 5 (1996) 173-208

4. P. Garber and S. Goldin-Meadow, "Gesture offers insight into problem-solving in adults and children," Cognitive Science 26 (2002) 817-831

5. W.-M. Roth and D. Lawless, "Scientific investigations, metaphorical gestures, and the emergence of abstract scientific concepts," Learning and Instruction 12 (2002) 285-304

6. R. B. Church and S. Goldin-Meadow, "The mismatch between gesture and speech as an index of transitional knowledge," Cognition 23 (1986) 43-71

7. The episode was videotaped. The video clip is posted at www.physics.umd.edu/perg/papers/scherr.

8. D. McNeill, Hand and Mind (1992), University of Chicago Press 